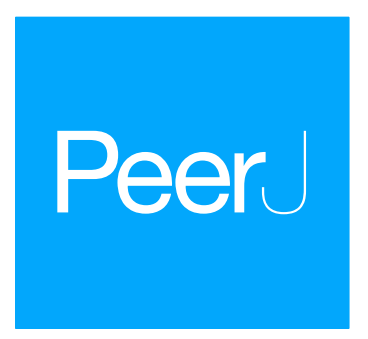

Submitted 29 March 2021 Accepted 5 August 2021 Published 25 August 2021

Corresponding authors

Yanyan Ping,

pingyanyan@hrbmu.edu.cn

Yihan Wang,

wangyihan@hrbmu.edu.cn

Academic editor

Howard Young

Additional Information and Declarations can be found on page 12

DOI 10.7717/peerj.12070

Copyright

2021 Lan et al.

Distributed under

Creative Commons CC-BY 4.0

OPEN ACCESS

\section{Prognostic impact of a lymphocyte activation-associated gene signature in GBM based on transcriptome analysis}

\author{
Yujia Lan*, Erjie Zhao*, Xinxin Zhang, Xiaojing Zhu, Linyun Wan, Suru A, \\ Yanyan Ping and Yihan Wang \\ Harbin Medical University, College of Bioinformatics Science and Technology, Harbin, China \\ These authors contributed equally to this work.
}

\section{ABSTRACT}

Background . Glioblastoma multiforme (GBM) is a highly, malignant tumor of the primary central nervous system. Patients diagnosed with this type of tumor have a poor prognosis. Lymphocyte activation plays important roles in the development of cancers and its therapeutic treatments.

Objective. We sought to identify an efficient lymphocyte activation-associated gene signature that could predict the progression and prognosis of GBM.

Methods. We used univariate Cox proportional hazards regression and stepwise regression algorithm to develop a lymphocyte activation-associated gene signature in the training dataset (TCGA, $n=525$ ). Then, the signature was validated in two datasets, including GSE16011 $(n=150)$ and GSE13041 $(n=191)$ using the Kaplan Meier method. Univariate and multivariate Cox proportional hazards regression models were used to adjust for clinicopathological factors.

Results. We identified a lymphocyte activation-associated gene signature (TCF3, IGFBP2, TYRO3 and NOD2) in the training dataset and classified the patients into high-risk and low-risk groups with significant differences in overall survival (median survival 15.33 months vs 12.57 months, $\mathrm{HR}=1.55,95 \%$ CI [1.28-1.87], log-rank test $P<0.001)$. This signature showed similar prognostic values in the other two datasets. Further, univariate and multivariate Cox proportional hazards regression models analysis indicated that the signature was an independent prognostic factor for GBM patients. Moreover, we determined that there were differences in lymphocyte activity between the high- and low-risk groups of GBM patients among all datasets. Furthermore, the lymphocyte activation-associated gene signature could significantly predict the survival of patients with certain features, including IDH-wildtype patients and patients undergoing radiotherapy. In addition, the signature may also improve the prognostic power of age.

Conclusions. In summary, our results suggested that the lymphocyte activationassociated gene signature is a promising factor for the survival of patients, which is helpful for the prognosis of GBM patients.

Subjects Bioinformatics, Immunology, Oncology, Medical Genetics, Computational Science Keywords Lymphocyte activation-associated gene signature, Glioblastoma multiforme (GBM), Prognostic biomarker, Lymphocyte activity, Overall survival 


\section{INTRODUCTION}

Glioblastoma multiforme (GBM) is classified as a grade IV diffuse glioma and is one of the most aggressive and lethal brain cancers. GBM has a high recurrence rate, typically originates in the cerebral hemispheres, and can quickly spread to the other parts of the brain (Alifieris $\&$ Trafalis, 2015; Batash, Asna \& Schaffer, 2017). IDH status is a classical marker of GBM, and patients with IDH mutations tended to have a better survival (Reuss, Mamatjan \& von Deimling, 2015; Burgenske, Yang \& Sarkaria, 2019; Aldape, Zadeh \& von Deimling, 2015). In previous studies, GBM patients can be divided into four distinct molecular subtypes based on gene expression profiling, including proneural, neural, classical and mesenchymal (Lee, Lee \& Lee, 2018; Liang et al. , 2005). The standard treatment for GBM is surgery, followed by radiation and chemotherapy (Alifieris \& Trafalis, 2015; Batash, Asna \& Schaffer, 2017; Wick et al., 2018). Recently, immunotherapies have been introduced for the treatment of GBM, especially for patients with EGFR mutations. However, the median survival time for GBM patients is only 15 months, which suggests that drug treatments may be ineffective for most of patients (Zanders, Svensson \& Bailey, 2019).

The lymphocyte activation is a set of processes where the lymphocytes are stimulated by specific antigens or nonspecific mitogens, which results in the synthesis of protein and production of lymphokines (Kay, 1991; Hodgkin, Chin \& Hasbold, 1997). These processes affect the proliferation and differentiation of various effector and memory cells. The effector cells will respond to antigens for the first time during the primary immune response. The memory cells can respond to a secondary immune response, which is known as immunological memory. Lymphocyte activation is destroyed in cancer, which is important for immunotherapy (Burugu, Dancsok \& Nielsen, 2018; Ware, 2008). Some lymphocyte activation-associated markers had been found to be associated with favorable survival and be effective for the treatments of patients, such as, lymphocyte activation gene 3 (LAG-3) (Wang, et al., 2018; Saleh et al., 2019; He et al., 2016). In addition, the lymphocyte activation combining with radiotherapy may be a novel treatment regimen for cancer patients (Akiyoshi, Tanaka \& Mori, 2019; Sato, Jeggo \& Shibata, 2019). Therefore, it is critical to develop a lymphocyte activation-associated prognostic signature of GBM to improve the treatment of patients.

In our study, a lymphocyte activation-associated gene signature was developed, which predicted the overall survival of GBM patients in training dataset. And we validated its prognostic power in another two datasets. The signature was found to be an independent prognostic factor after adjusting for other clinicopathologic factors. Moreover, this signature not only predicted the survival of patients with IDH wild-type glioblastoma, but also patients after radiotherapy. These findings indicated the signature may serve as an effective prognostic biomarker for patients with GBM.

\section{MATERIALS AND METHODS \\ Datasets description}

We downloaded three gene expression datasets (TCGA, GSE16011 (Gravendeel et al. , 2009) and GSE13041 (Lee, Scheck \& Nelson, 2008)) from The Cancer Genome Atlas (TCGA) 
Table 1 Clinical and pathological characteristics of GBM patients in TCGA.

\begin{tabular}{lll} 
Clinical features & Category & GBM, $\boldsymbol{n}=\mathbf{5 2 5}$ \\
\hline Gender & Female & 205 \\
& Male & 320 \\
Age & Median (range) & $59(10-89)$ \\
Classical Subtype & Classical & 144 \\
& Mesenchymal & 155 \\
& Neural & 83 \\
Survival status & Proneural & 99 \\
& Alive & 75 \\
Follow-up from samples (months) & Deceased & 449 \\
\hline
\end{tabular}

Table 2 Clinical and pathological characteristics of GBM patients in GSE13041 and GSE16011.

\begin{tabular}{llll} 
Clinical features & Category & $\begin{array}{l}\text { GSE13041 } \\
(\boldsymbol{n}=\mathbf{1 9 1})\end{array}$ & $\begin{array}{l}\text { GSE16011 } \\
(\boldsymbol{n}=\mathbf{1 5 0})\end{array}$ \\
\hline Gender & Female & 74 & 47 \\
& Male & 117 & 103 \\
Age & Median (range) & $54(18-86)$ & $55.44(14.38-80.65)$ \\
Survival status & Alive & 15 & 3 \\
& Deceased & 176 & 147 \\
Follow-up from samples (months) & Median (range) & $12.97(0.23-111.77)$ & $8.7(0.24-150.72)$ \\
\hline
\end{tabular}

portal (https://portal.gdc.cancer.gov) and Gene Expression Omnibus (GEO) (Edgar, Domrachev \& Lash, 2002; Barrett et al., 2013), which contained patient outcome and clinicopathological factors. The gene expression profile of each dataset was normalized by replacing the expression level e with $\log 2(\mathrm{e}+1)$. The patients without matched clinical information were excluded. Consequently, a total of $866 \mathrm{GBM}$ patients (525 GBM patients from TCGA, 191 GBM patients from GSE13041 and 150 GBM patients from GSE16011) were included in this study. The TCGA dataset was treated as a training dataset, and another two datasets (GSE16011 and GSE13041) were used as independent validation cohorts. In the training dataset, the median survival time of GBM patients was 12.4 months (ranging from 0.1 to 127.5 months). And there were 144 classical, 155 mesenchymal, 83 neural, and 99 proneural GBM samples. In Table 1 we summarized clinicopathological data belonging to GBM patients in the TCGA dataset. The median survival of GBM patients was 13.0 months (ranging from 0.2 to 111.8 months) and 8.7 months (ranging from 0.2 to 150.7 months) in the validation datasets, respectively (Table 2 ).

\section{Identifying the lymphocyte activation-associated gene signature} Differential expression analysis was performed based on transcriptome profile of GBM patients by the "limma" $\mathrm{R}$ package in the training dataset. Genes with the cutoff criteria of $\mid \log 2$-fold change $\mid \geq 1$ and FDR $<0.000001$ between tumor and normal tissues were regarded as differentially expressed genes (DEGs). Using the univariate Cox proportional 
hazards regression algorithm, we retained the DEGs that were strongly associated with the survival of patients with GBM $(P<0.05)$. We focused on the prognostic genes that participated in the lymphocyte activation process. The lymphocyte activation-related genes were collected by GO terms in Gene Ontology (Ashburner et al., 2000; Gene Ontology, 2021). Then, we further selected genes associated with overall survival by stepwise regression analysis in the training dataset. Ultimately, we kept genes with the smallest Akaike information criteria (AIC) value to construct the prognostic model. There were four genes (TCF3, IGFBP2, TYRO3 and NOD2) that were identified in our study (Fig. S1). Finally, a risk score model was developed based on the gene expression weighted by regression coefficients of univariable Cox regression: Risk score $=(-0.6246210 \times$ expression level of TCF3 $)+(0.2992 \times$ expression level of IGFBP2 $)+(0.2421068 \times$ expression level of TYRO3 $)+(0.2155469 \times$ expression level of NOD2). Based on this model, the patients were classified into the high- and low-risk groups by the median risk score in the training dataset.

\section{Statistical analysis}

In the survival analysis, we analyzed only overall survival as the end point. The survival differences between the two groups were visualized by Kaplan-Meier analysis and were compared by the log-rank test. The prognostic value of the lymphocyte activation-associated gene signature was estimated by univariate and multivariate Cox proportional hazards regression models. The Cox proportional hazards regression model was used to calculate hazard ratio (HR) and 95\% confidence intervals (CI). The $P$-values smaller than 0.05 were considered to be statistically significant. We obtained gene signatures (that is cell markers) of lymphocytes (including natural killer cells, T cells and B cells) from the CIBERSORT web portal (Newman et al., 2015) and MCP-counter web portal (Becht et al., 2016). We combined information from these two sources to get gene signatures of lymphocytes. The lymphocyte activity of each patient was calculated by single sample gene set enrichment analysis (ssGSEA) (Barbie et al., 2009) based on the gene signatures of lymphocytes. Each ssGSEA enrichment score represents the degree to which the genes in a particular gene set are coordinately regulated within a sample. In our study, the gene signature of lymphocytes means the gene set of ssGSEA and the lymphocyte activity means the enrichment score. The concordance index (C-index) was used to compare the prognostic efficacy among this signature, age, sex, and the combined model using a logistic regression with the aforementioned three variables, and the significant $p$-value were calculated by rcorrp.cens function in Hmisc package. R software (http://www.r-project.org) was used to perform all of the statistical analyses, version 3.5.1 (Packages: survival, survminer, limma, Hmisc, ggplot2, GSVA).

\section{RESULTS}

\section{Development of a prognostic signature related to lymphocyte activation}

The gene expression profile and clinical information of GBM patients were obtained from TCGA, which were treated as the training dataset. There were 525 tumor samples and 

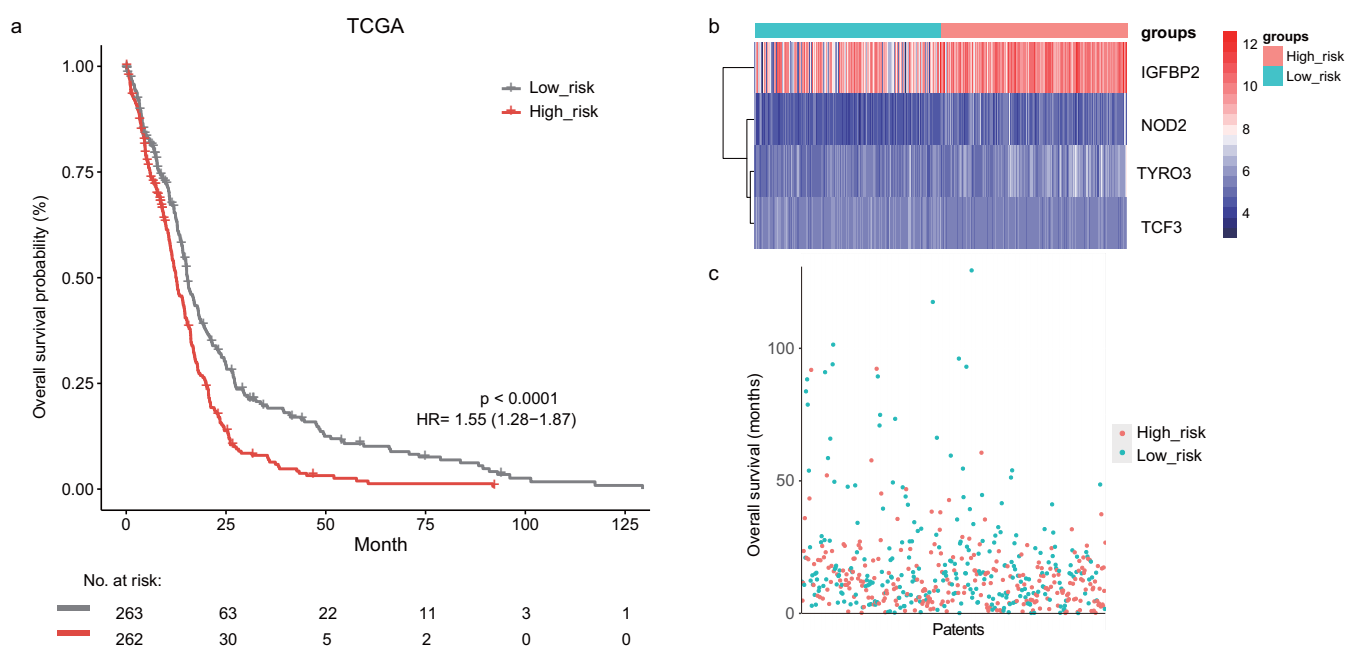

Figure 1 The survival analysis of the lymphocyte activation-associated gene signature in training set (TCGA, $\boldsymbol{n}=525$ ). (A) Kaplan-Meier curves of overall survival for the four gene signature. The patients were divided into high-risk (red) and low-risk group (grey). (B) The heatmap showing expression profiles of four genes in the signature. (C) The survival status and time distribution of the GBM patients.

Full-size DOI: 10.7717/peerj.12070/fig-1

10 normal samples. The tumor and normal samples came from different samples. We identified 2,364 differentially expressed genes between tumor and normal samples by using the limma package (FDR $<0.000001$ and $\mid \log 2$-fold change $\mid \geq 1$ ), including 933 up-regulated and 1,431 down-regulated genes (Fig. S2). We obtained 173 differentially expressed genes that could be used for prognosis by univariate Cox proportional hazards regression. Genes were subjected to the lymphocyte activation function, which was obtained from Gene Oncology (GO). This resulted in 12 lymphocyte activation-associated genes that were used for subsequent analysis. Finally, we obtained four genes (TCF3, IGFBP2, TYRO3 and NOD2) that were significantly related to overall survival using a stepwise regression algorithm. We used the lymphocyte activation-associated gene signature to calculate each patient's risk score based the four genes' expression levels weighted by regression coefficients in the univariate Cox proportional hazards regression analysis (Fig. S1). Positive coefficients of IGFBP2, TYRO3, and NOD2 (0.2992, 0.2421068 and 0.2155469 ) suggested that their expression was associated with poor prognosis in GBM patients, while the negative coefficient of TCF3 $(-0.6246210)$ indicated it was associated with better survival.

In the training dataset, GBM patients were divided into high-risk $(n=262)$ and low-risk ( $n=263$ ) groups according to the median risk score (2.752) of the lymphocyte activationassociated gene signature. This score was used as the cutoff. Patients within high-risk group had significantly shorter overall survival time than those within low-risk group (median survival 15.3 months $v s 12.6$ months, $\mathrm{HR}=1.55,95 \%$ CI [1.28-1.87], log-rank test $P<$ 0.001 , Fig. 1A). The gene expression and survival time distributions suggested that patients in the high-risk group had higher IGFBP2 expression (Fig. 1B). The low-risk patients tended to have a longer survival time (Fig. 1C). 

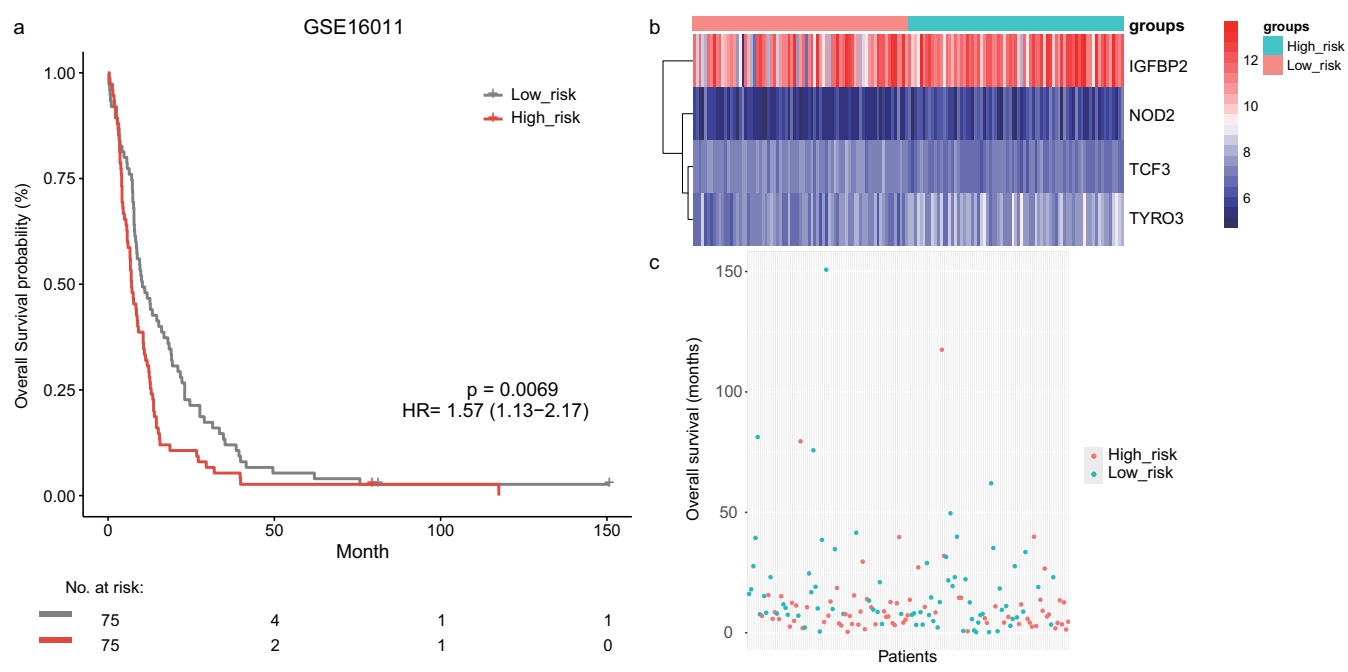

Figure 2 The survival analysis of the lymphocyte activation-associated gene signature in GSE16011 $(n=150)$. (A) Kaplan-Meier curves of overall survival for the four-gene signature with the median value as the cutoff. (B) The expression profiles of the four genes in the signature. (C) The distribution of the GBM patients' overall survival status.

Full-size DOI: 10.7717/peerj.12070/fig-2

\section{Prognostic value of the lymphocyte activation-associated gene signature in validation sets}

Next, we validated the prognostic value of the lymphocyte activation-associated gene signature in another two external validation datasets, GSE13041 $(n=191)$ and GSE16011 $(n=150)$. Each patient's risk score was calculated with the same formula as that used in the training set. The patients were divided into the high- and low-risk groups according to the median cutoff determined for each dataset. In GSE16011, survival time of patients in the high-risk group were significantly shorter than those in the low-risk group (median survival 7.1 months $v s 10.3$ months, $\mathrm{HR}=1.57,95 \% \mathrm{CI}$ [1.13-2.17], log-rank test $P=0.069$, Fig. 2A). IGFBP2 and TYRO3 had a relatively higher expression in the high-risk group (Fig. 2B). We found the low-risk patients presented a trend of longer survival time in GSE16011 (Fig. 2C). Moreover, the signature was found to predict the survival of patients with GBM in GSE13041 (median survival of high-risk patients 12.0 months vs median survival of low-risk patients 14.5 months, $\mathrm{HR}=1.42,95 \%$ CI [1.05-1.92], log-rank test $P=0.02$, Figs. 3A-3C) with statistical significance.

\section{The signature independently predicted overall survival of GBM patients}

We used univariate and multivariable Cox regression analysis after adjusting for other clinicopathologic factors to assess whether the lymphocyte activation-associated gene signature was an independent prognostic biomarker in GBM. The covariables included age, sex, IDH status, subtypes, and the prognostic signature. We found that the signature $(\mathrm{HR}=1.46,95 \% \mathrm{CI}[1.16-1.83], P=0.001)$, age $(\mathrm{HR}=1.03,95 \% \mathrm{CI}[1.02-1.04], P<$ $0.001)$, sex (HR $=1.30,95 \% \mathrm{CI}[1.04-1.63], P=0.02)$ and proneural types $(\mathrm{HR}=1.64$, 

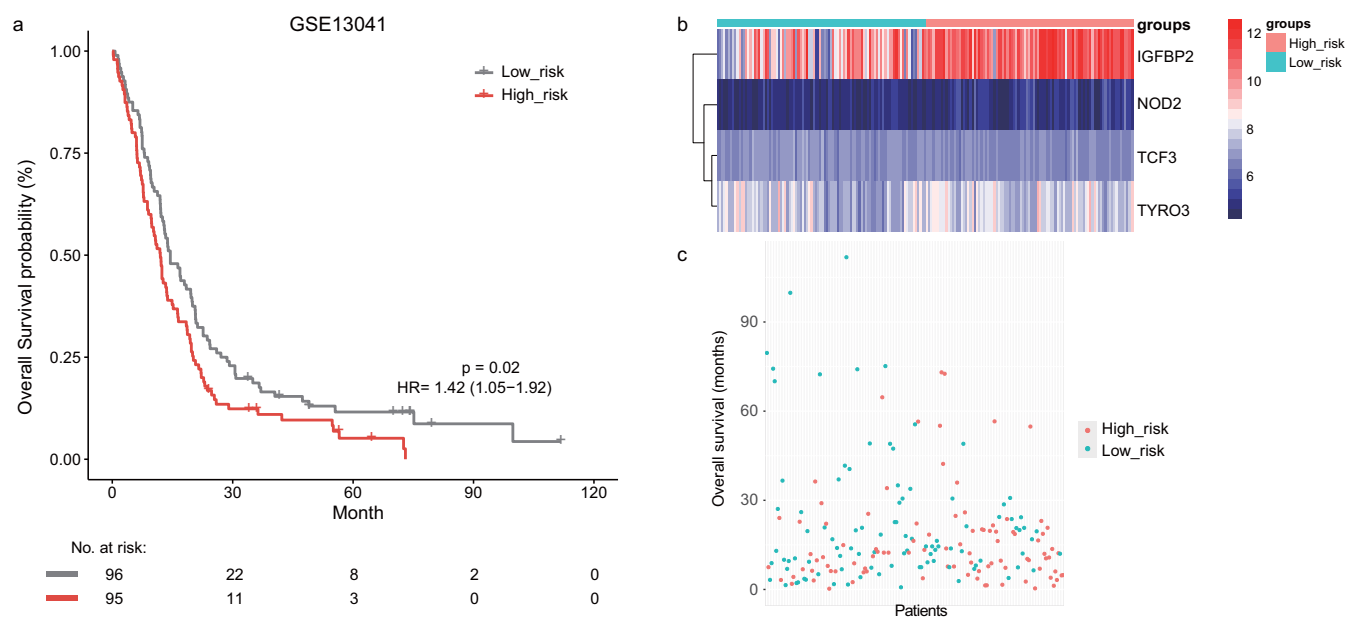

Figure 3 The survival analysis of the lymphocyte activation-associated gene signature in GSE13041 $(n=191)$. (A) Kaplan-Meier curves of overall survival for the four-gene signature with the median value as the cutoff. (B) The expression profiles of the four genes in the signature. (C) The distribution of the GBM patients' overall survival status.

Full-size DOI: 10.7717/peerj.12070/fig-3

Table 3 Multivariate analysis for the lymphocyte activation-associated gene signature of overall survival in TCGA.

\begin{tabular}{|c|c|c|c|c|c|c|c|}
\hline \multirow[t]{2}{*}{ Variables } & & \multicolumn{3}{|c|}{ Univariate } & \multicolumn{3}{|c|}{ Multivariate } \\
\hline & & HR & 95\% CI & $P$ value & HR & $95 \% \mathrm{CI}$ & $P$ value \\
\hline Age & & 1.032 & $1.025-1.04$ & $<0.001^{*}$ & 1.028 & $1.018-1.037$ & $<0.001^{*}$ \\
\hline Sex & Male vs Female & 1.241 & $0.993-1.551$ & 0.057 & 1.3 & $1.035-1.634$ & $0.024^{*}$ \\
\hline IDH status & Mutation $v s$ Wild type & 0.353 & $0.229-0.544$ & $<0.001^{*}$ & 0.831 & $0.396-1.742$ & 0.623 \\
\hline Signature & High_risk $v s$ Low_risk & 1.588 & $1.275-1.979$ & $<0.001^{*}$ & 1.455 & $1.159-1.828$ & $0.001^{*}$ \\
\hline \multirow[t]{4}{*}{ Subtypes } & G-CIMP vs Classical & 0.369 & $0.2271-0.599$ & $<0.001^{*}$ & 0.701 & $0.319-1.544$ & 0.378 \\
\hline & Mesenchymal vs Classical & 1.169 & $0.876-1.56$ & 0.289 & 1.117 & $0.835-1.494$ & 0.455 \\
\hline & Neural vs Classical & 1.091 & $0.788-1.509$ & 0.6 & 1.177 & $0.848-1.633$ & 0.331 \\
\hline & Proneural $v s$ Classical & 1.314 & $0.954-1.808$ & 0.094 & 1.638 & $1.184-2.268$ & $0.003^{*}$ \\
\hline
\end{tabular}

Notes.

${ }^{\star}$ Significant $P$ values are labeled with ${ }^{*}(P<0.05)$.

95\% CI [1.18-2.27], $P=0.003$ ) independently predicted a worse OS for GBM patients in the training dataset (Table 3). In the validation dataset GSE16011, we also found that the lymphocyte activation-associated gene signature had independently prognostic value (HR $=1.36,95 \%$ CI $[1.35-1.98], P=0.029$, Table 4$)$. This signature independently predicted the survival of GBM patients, with marginal significance (HR $=1.33,95 \%$ CI [0.98-1.81], $P=0.06$, Table S1). These findings revealed that the prognostic ability of the lymphocyte activation-associated gene signature was independent of the clinicopathological factors for OS in GBM. 


\begin{tabular}{|c|c|c|c|c|c|c|c|}
\hline \multirow[t]{2}{*}{ Variables } & & \multicolumn{3}{|c|}{ Univariate } & \multicolumn{3}{|c|}{ Multivariate } \\
\hline & & HR & $95 \% \mathrm{CI}$ & $P$ value & HR & 95\% CI & $P$ value \\
\hline Age & & 1.039 & $1.024-1.054$ & $<0.001^{*}$ & 1.031 & $1.024-1.039$ & $<0.001^{*}$ \\
\hline Sex & Male vs Female & 0.809 & $0.544-1.204$ & 0.296 & 0.928 & $0.62-1.391$ & 0.719 \\
\hline IDH status & Mutation $v s$ wild type & 0.579 & $0.367-0.915$ & $0.019^{*}$ & 0.809 & $0.493-1.326$ & 0.4 \\
\hline Signature & High_risk $v s$ Low_risk & 1.554 & $1.284-1.88$ & $<0.001^{*}$ & 1.359 & $1.346-1.979$ & $0.029^{*}$ \\
\hline
\end{tabular}

Notes.

${ }^{\star}$ Significant $P$ values are labeled with ${ }^{*}(P<0.05)$.

\section{The differences in lymphocyte activity between the high- and low-risk groups of patients}

The GBM patients were divided into low- and high-risk groups based on their lymphocyte activation-associated gene signature. We then compared the lymphocyte activity in these two groups. Lymphocytes include natural killer cells, T cells and B cells. In TCGA, five types of lymphocytes showed significant differences in activity between high- and low-risk patients by Wilcoxon ranked sum test $(P<0.05)$. These lymphocytes were memory B cells, naive B cells, naive CD4 T cells, follicular helper T cells and regulatory T cells (Fig. 4A and Fig. S3). And the lymphocyte activity of high-risk patients was significantly lower than in low-risk patients. Similar phenomena were observed in the GSE13041 dataset (Fig. 4B). However, activated CD4 memory T cells, resting CD4 memory T cells, CD8 T cells and gamma delta $\mathrm{T}$ cells showed significant differences between the two groups. There were three types of cells that showed different activity between low- and high-risk patients in the GSE16011 dataset $(P<0.05$, Fig. S4). These findings suggested that lymphocyte activity was significantly different between the high- and low-risk GBM patients.

\section{Stratification analysis of the lymphocyte activation-associated gene signature}

The IDH mutation is one of the most critical genomic alterations in GBM. The IDH mutation means a somatic mutation in IDH1 in our study. IDH-wildtype GBM patients had a shorter survival time than those with the IDH-mutation. The lymphocyte activationassociated gene signature significantly predicted the overall survival of IDH-wildtype GBM patients by log-rank test $(P=0.0043)$ but it did not predict the survival of IDH-mutation patients (Fig. 5A). The IDH-wildtype patients were divided into high-risk and low-risk groups using the same cutoff in the training dataset. The high-risk group of IDH-wildtype patients had significantly shorter OS than those in the low-risk group (median survival 12.9 months vs 14.9 months, $\mathrm{HR}=1.39,95 \%$ CI [1.11-1.74], $P=0.0043$, Fig. 5A). The same phenomenon was also found in the GSE16011 dataset. The high-risk patients showed worse OS than the low-risk patients (median survival 7.1 months $v s 10.7$ months, $\mathrm{HR}=$ 1.95, 95\% CI [1.25-3.02], $P=0.0025$ ) among IDH-wildtype patients (Fig. 5B).

We next explored whether the lymphocyte activation-associated gene signature was effective for GBM patients within four transcriptome subtypes (proneural, neural, classical, and mesenchymal) established by Verhaak et al. using Kaplan-Meier survival analysis. 

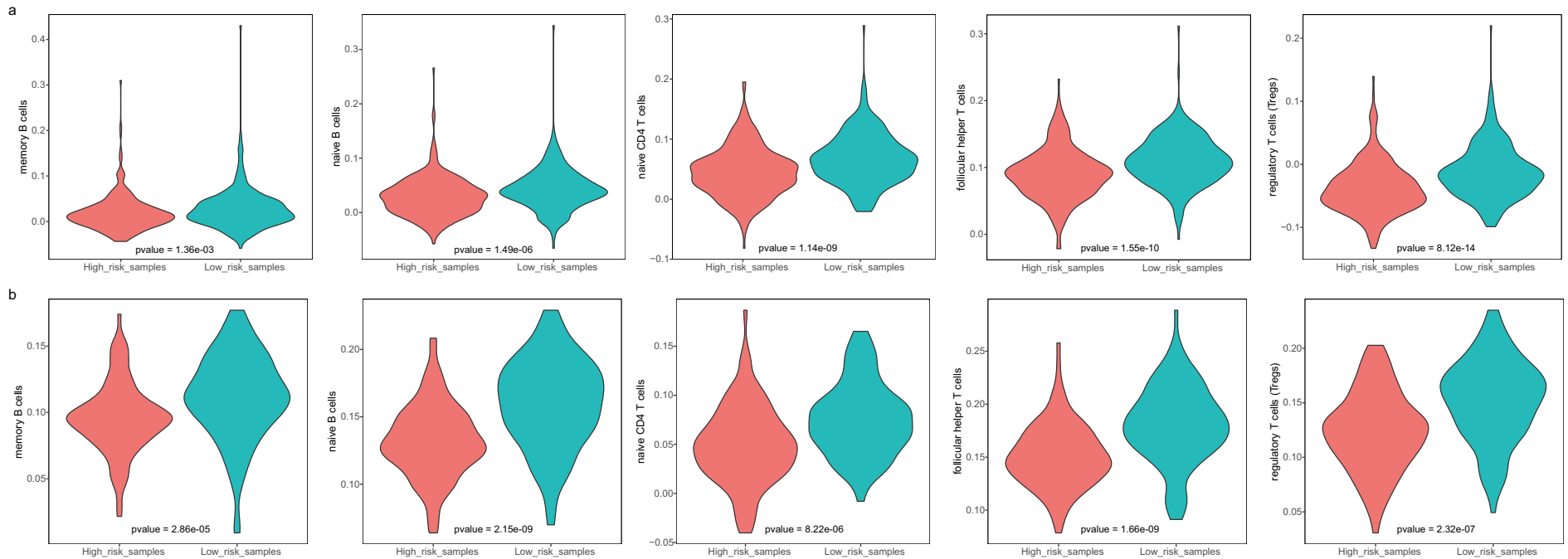

Figure 4 The cell activity of lymphocytes (memory B cells, native B cells, native CD4 T cells, follicular helper T cells and the regulatory T cells) in TCGA (A) and the GSE13041 (B) dataset.

Full-size DOI: 10.7717/peerj.12070/fig-4

The signature significantly predicted the overall survival of patients with the mesenchymal and proneural subtypes (log-rank test $P$ value $=0.013$ for mesenchymal subtypes and $P$ value $=0.041$ for proneural subtypes, Fig. 6). The patients in the high-risk groups had a shorter survival time than those in the low-risk groups (median survival 11.8 months vs 15.3 months for mesenchymal subtypes and 9 months $v s 13.2$ months for proneural subtypes, respectively). In addition, the signature did not have the prognostic ability for patients with neural and classical subtypes (Fig. S5).

We also explored whether radiotherapy was an effective treatment for GBM patients. We observed that high risk score significantly predicted a poor OS for GBM patients undergoing radiotherapy in the GSE16011 (median survival 8.76 months vs 14.64 months, $\mathrm{HR}=1.86,95 \% \mathrm{CI}[1.27-2.72], P=0.0011)$ and GSE13041 datasets (median survival 6.03 months $v s 12.53$ months, $\mathrm{HR}=3.18,95 \% \mathrm{CI}[1.13-8.94], P=0.022$, Fig. 7).

\section{Comparison of prognostic power between clinical factors and the signature}

To evaluate the prognostic performance of the prognostic signature, we performed C-index calculation of clinical factors (age and sex) and the signature in three datasets (Harrell $\mathrm{Jr}$ et al, 1982; Pencina \& D'Agostino, 2004). We constructed another prognostic model by combining our signature with clinical factors. A higher C-index indicated a superior prognostic value of signature (Wu, Yuan \& Liu, 2019; Li, Cui \& Li, 2017). There were significant differences found between age and the signature $(P<0.05)$ and between age and sex. The clinical factors for age in combination with the signature showed a higher C-index (0.646 for TCGA, 0.665 for GSE16011 and 0.600 for GSE13041) than age or histological grade alone (0.645 for TCGA, 0.659 for GSE16011 and 0.595 for GSE13041, Table S2), which were statistically significant $(P<0.05)$. These results suggested that this 
a



b

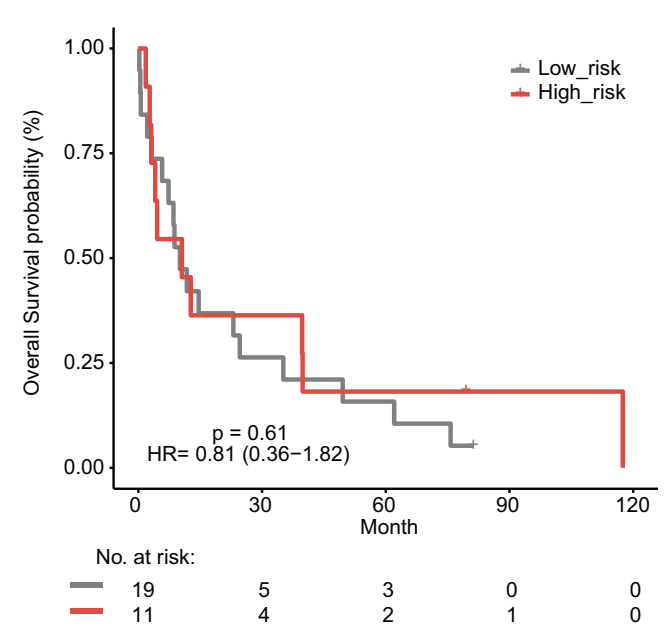

TCGA.IDH.wildtype

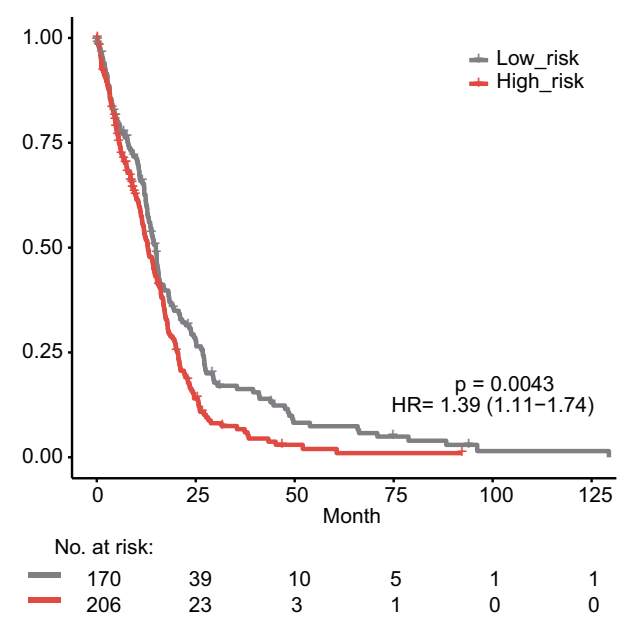

GSE16011.IDH.wildtype

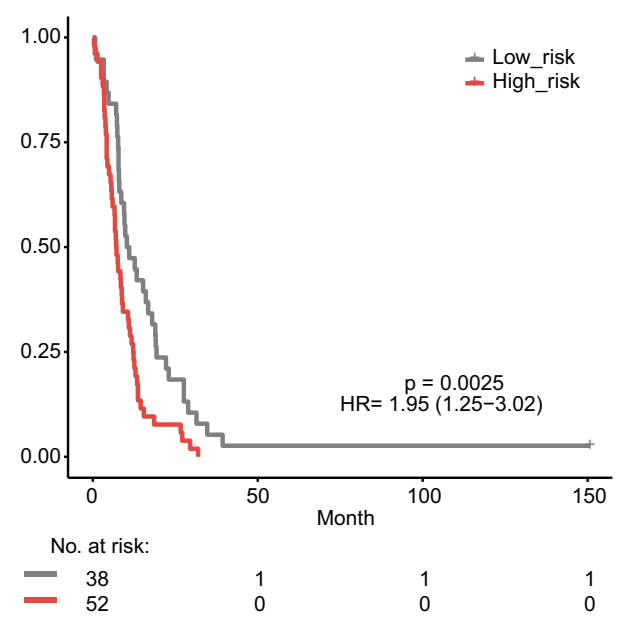

Figure 5 Kaplan-Meier analysis estimates of overall survival in TCGA (A) and the GSE16011 (B) datasets according to the IDH1 mutation status.

Full-size DOI: 10.7717/peerj.12070/fig-5

lymphocyte activation-associated gene signature could add complementary value to known clinical factors.

\section{DISCUSSION}

In this study, we identified a lymphocyte activation-associated gene signature that predicted shorter overall survival of patients with GBM. The prognostic power of this signature was evaluated by univariate and multivariate Cox proportional hazards regression models analysis using three datasets $(\mathrm{HR}>1)$. Our findings indicated that the gene signature was an unfavorable factor in GBM. Moreover, the high-risk and low-risk patients, which were separated by the signature, showed significant differences in immune cell activity. This signature was found to be an independent prognostic factor after adjusting for certain clinicopathological factors. In addition, the lymphocyte activation-associated gene 
TCGA.Mesenchymal

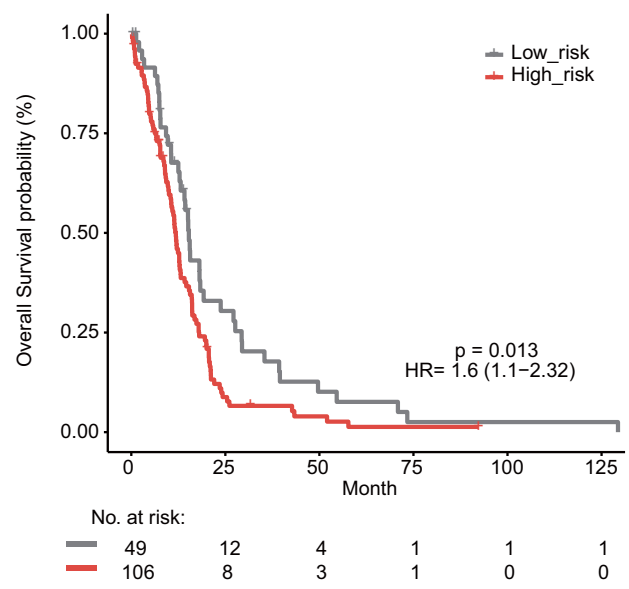

TCGA.Proneural

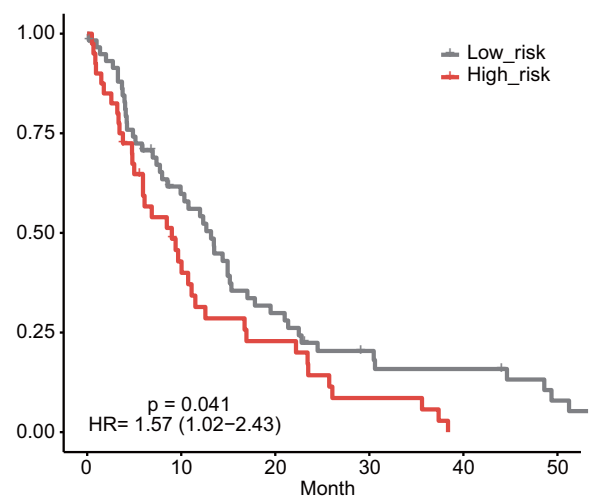

Figure 6 Survival analysis for GBM patients according to TCGA mesenchymal and proneural subtypes.

Full-size DOI: 10.7717/peerj.12070/fig-6

GSE16011.Radiotherapy.yes



GSE13041.Radiotherapy.yes

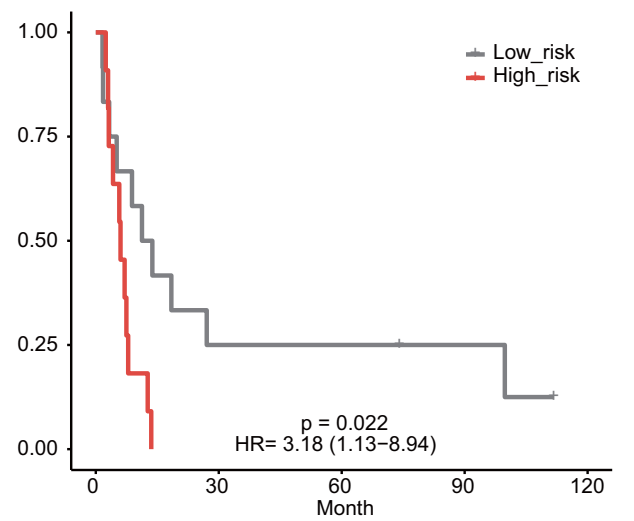

$\begin{array}{lllll}\text { No. at risk: } & & & & \\ -12 & 3 & 3 & 2 & 0 \\ -11 & 0 & 0 & 0 & 0\end{array}$

Figure 7 The signature predicts cancer patient outcome after radiotherapy.

Full-size DOI: 10.7717/peerj.12070/fig-7

signature also predicted the survival of patients with an IDH wild-type variant and for patients after radiotherapy treatment.

We developed a prognostic signature that included four genes (TCF3, IGFBP2, TYRO3 and NOD2). IGFBP2 was one of the insulin-like growth factor binding proteins (IGFBPs), which are proteins binding to Insulin-like growth factors. IGFBP2 expression increased in peripheral blood mononuclear cells and participated in lymphocyte proliferation (Hettmer et al., 2005). In various autoimmune diseases, IGFBP2 can be as potential biomarker and therapeutic target (Ding \& Wu, 2018). Moreover, IGFBP2 expression predicted the survival in GBM patients (Yuan et al., 2019; Cai et al., 2018). NOD2 played important roles in 
the pathogenesis of some diseases, such as, oral lichen planus (Ahn et al., 2020), Crohn's disease (Niess et al., 2012), and inflammatory bowel disease (Franchi et al., 2008). NOD2 expression is higher in activated/memory CD4+ T cells (Zanello et al., 2013) and was found to be a new diagnostic and treatment target for disease (Ahn et al., 2020). A prognostic model was constructed by 9 immune genes, including NOD2, which predicted the shorter survival of GBM patients (Liang, Chai \& Wang, 2020).

Another marker, an important transcription factor TCF3 played a role in germinal center B - cell development and promoted cell growth, which contributed to proliferative phenotype in Burkitt lymphoma (Richter et al., 2012; Dave et al., 2006; Sakata-Yanagimoto et al., 2014). In addition, TCF3 can regulate B-cell-restricted genes through E-box motifs (Barbie et al., 2009). It can promote survival of Burkitt's lymphoma cells by activating B-cell receptor signaling and PI3K signaling pathways and by modulating cell cycle regulators (Schmitz et al., 2012). Also, TCF3 promotes the survival in lymphoid cells (Sakata-Yanagimoto et al., 2014). TYRO3 is a protein-coding gene, which participates in ERK signaling pathway. The higher level of TYRO3 expression is associated with decreased overall survival in patients with colorectal, hepatocellular, and breast cancers (Smart et al., 2018). Although the latter two genes have not been directly shown to be associated with GBM, the prognostic efficacy of these two genes should be verified in future studies.

In conclusion, we developed a lymphocyte activation-associated gene signature with prognostic power and offered new insights for the treatments of GBM. However, more data are needed to test the prognostic value of the signature before applying it to clinical management. A larger study is needed to confirm that the signature can accurately predict the prognostic benefits for GBM patients.

\section{ADDITIONAL INFORMATION AND DECLARATIONS}

\section{Funding}

This work was supported by the Heilongjiang Postdoctoral Foundation (Grant No. LBHZ19082) and the Special funds for the construction of higher education in Heilongjiang Province (Grant No. UNPYSCT-2018068). There was no additional external funding received for this study. The funders had no role in study design, data collection and analysis, decision to publish, or preparation of the manuscript.

\section{Grant Disclosures}

The following grant information was disclosed by the authors:

Heilongiiang Postdoctoral Foundation: LBH-Z19082.

Heilongjiang Province: UNPYSCT-2018068.

\section{Competing Interests}

The authors declare there are no competing interests. 


\section{Author Contributions}

- Yujia Lan conceived and designed the experiments, performed the experiments, analyzed the data, prepared figures and/or tables, authored or reviewed drafts of the paper, and approved the final draft.

- Erjie Zhao and Xinxin Zhang performed the experiments, analyzed the data, prepared figures and/or tables, and approved the final draft.

- Xiaojing Zhu, Linyun Wan and Suru A performed the experiments, prepared figures and/or tables, and approved the final draft.

- Yanyan Ping and Yihan Wang conceived and designed the experiments, authored or reviewed drafts of the paper, and approved the final draft.

\section{Data Availability}

The following information was supplied regarding data availability:

The data are available at TCGA (TCGA-GBM) and NCBI GEO (GSE16011 and GSE13041).

\section{Supplemental Information}

Supplemental information for this article can be found online at http://dx.doi.org/10.7717/ peerj.12070\#supplemental-information.

\section{REFERENCES}

Ahn MY, Kang JK, Kwon SM, Yoon HE, Yoon JH. 2020. Expression of nucleotidebinding oligomerization domain 1 and 2 in oral lichen planus. Journal of Dental Sciences 15(1):1-8 DOI 10.1016/j.jds.2019.12.005.

Akiyoshi T, Tanaka N, Kiyotani K, Gotoh O, Yamamoto N, Oba K, Fukunaga Y, Ueno M, Mori S. 2019. Immunogenomic profiles associated with response to neoadjuvant chemoradiotherapy in patients with rectal cancer. British Journal of Surgery 106(10):1381-1392 DOI 10.1002/bjs.11179.

Aldape K, Zadeh G, Mansouri S, Reifenberger G, von Deimling A. 2015. Glioblastoma: pathology molecular mechanisms and markers. Acta Neuropathologica 129(6):829-848 DOI 10.1007/s00401-015-1432-1.

Alifieris C, Trafalis DT. 2015. Glioblastoma multiforme: pathogenesis and treatment. Pharmacology and Therapeutics 152:63-82 DOI 10.1016/j.pharmthera.2015.05.005.

Ashburner M, Ball CA, Blake JA, Botstein D, Butler H, Cherry JM, Davis AP, Dolinski K, Dwight SS, Eppig JT, Harris MA, Hill DP, Issel-Tarver L, Kasarskis A, Lewis S, Matese JC, Richardson JE, Ringwald M, Rubin GM, Sherlock G. 2000. Gene ontology: tool for the unification of biology. The gene ontology consortium. Nature Genetics 25(1):25-29.

Barbie DA, Tamayo P, Boehm JS, Kim SY, Moody SE, Dunn IF, Schinzel AC, Sandy P, Meylan E, Scholl C, Frohling S, Chan EM, Sos ML, Michel K, Mermel C, Silver SJ, Weir BA, Reiling JH, Sheng Q, Gupta PB, Wadlow RC, Le H, Hoersch S, Wittner BS, Ramaswamy S, Livingston DM, Sabatini DM, Meyerson M, Thomas 
RK, Lander ES, Mesirov JP, Root DE, Gilliland DG, Jacks T, Hahn WC. 2009. Systematic RNA interference reveals that oncogenic KRAS-driven cancers require TBK1. Nature 462(7269):108-112 DOI 10.1038/nature08460.

Barrett T, Wilhite SE, Ledoux P, Evangelista C, Kim IF, Tomashevsky M, Marshall KA, Phillippy KH, Sherman PM, Holko M, Yefanov A, Lee H, Zhang N, Robertson CL, Serova N, Davis S, Soboleva A. 2013. NCBI GEO: archive for functional genomics datasets-update. Nucleic Acids Research 41(Database issue):D991-D995.

Batash R, Asna N, Schaffer P, Francis N, Schaffer M. 2017. Glioblastoma multiforme diagnosis and treatment; recent literature review. Current Medicinal Chemistry 24(27):3002-3009.

Becht E, Giraldo NA, Lacroix L, Buttard B, Elarouci N, Petitprez F, Selves J, LaurentPuig P, Sautes-Fridman C, Fridman WH, De Reynies A. 2016. Estimating the population abundance of tissue-infiltrating immune and stromal cell populations using gene expression. Genome Biology 17(1):218 DOI 10.1186/s13059-016-1070-5.

Burgenske DM, Yang J, Decker PA, Kollmeyer TM, Kosel ML, Mladek AC, Caron AA, Vaubel RA, Gupta SK, Kitange GJ, Sicotte H, Youland RS, Remonde D, Voss JS, Fritcher EGB, Kolsky KL, Ida CM, Meyer FB, Lachance DH, Parney IJ, Kipp BR, Giannini C, Sulman EP, Jenkins RB, Eckel-Passow JE, Sarkaria JN. 2019. Molecular profiling of long-term IDH-wildtype glioblastoma survivors. Neuro-Oncology 21(11):1458-1469 DOI 10.1093/neuonc/noz129.

Burugu S, Dancsok AR, Nielsen TO. 2018. Emerging targets in cancer immunotherapy. Seminars in Cancer Biology 52(Pt 2)):39-52.

Gene Ontology C. 2021. The Gene Ontology resource: enriching a GOld mine. Nucleic Acids Research 49(D1):D325-D334 DOI 10.1093/nar/gkaa1113.

Cai J, Chen Q, Cui Y, Dong J, Chen M, Wu P, Jiang C. 2018. Immune heterogeneity and clinicopathologic characterization of IGFBP2 in 2447 glioma samples. Oncoimmunology 7(5):e1426516 DOI 10.1080/2162402X.2018.1426516.

Dave SS, Fu K, Wright GW, Lam LT, Kluin P, Boerma EJ, Greiner TC, Weisenburger DD, Rosenwald A, Ott G, Muller-Hermelink HK, Gascoyne RD, Delabie J, Rimsza LM, Braziel RM, Grogan TM, Campo E, Jaffe ES, Dave BJ, Sanger W, Bast M, Vose JM, Armitage JO, Connors JM, Smeland EB, Kvaloy S, Holte H, Fisher RI, Miller TP, Montserrat E, Wilson WH, Bahl M, Zhao H, Yang L, Powell J, Simon R, Chan WC, Staudt LM. 2006. P. Lymphoma/leukemia molecular profiling, molecular diagnosis of Burkitt's lymphoma. New England Journal of Medicine 354(23):2431-2442 DOI 10.1056/NEJMoa055759.

Ding H, Wu T. 2018. Insulin-like growth factor binding proteins in autoimmune diseases. Frontiers in Endocrinology 9:499 DOI 10.3389/fendo.2018.00499.

Edgar R, Domrachev M, Lash AE. 2002. Gene expression omnibus: NCBI gene expression and hybridization array data repository. Nucleic Acids Research 30(1):207-210 DOI 10.1093/nar/30.1.207.

Harrell Jr FE, Califf RM, Pryor DB, Lee KL, Rosati RA. 1982. Evaluating the yield of medical tests. Journal of the American Medical Association 247(18):2543-2546 DOI 10.1001/jama.1982.03320430047030. 
Franchi L, Park JH, Shaw MH, Marina-Garcia N, Chen G, Kim YG, Nunez G. 2008. Intracellular NOD-like receptors in innate immunity, infection and disease. Cellular Microbiology 10(1):1-8.

Gravendeel LA, Kouwenhoven MC, Gevaert O, De Rooi JJ, Stubbs AP, Duijm JE, Daemen A, Bleeker FE, Bralten LB, Kloosterhof NK, De Moor B, Eilers PH, van der Spek PJ, Kros JM, Sillevis Smitt PA, Van den Bent MJ, French PJ. 2009. Intrinsic gene expression profiles of gliomas are a better predictor of survival than histology. Cancer Research 69(23):9065-9072 DOI 10.1158/0008-5472.CAN-09-2307.

He Y, Rivard CJ, Rozeboom L, Yu H, Ellison K, Kowalewski A, Zhou C, Hirsch FR. 2016. Lymphocyte-activation gene- 3, an important immune checkpoint in cancer. Cancer Science 107(9):1193-1197 DOI 10.1111/cas.12986.

Hettmer S, Dannecker L, Foell J, Elmlinger MW, Dannecker GE. 2005. Effects of insulin-like growth factors and insulin-like growth factor binding protein-2 on the in vitro proliferation of peripheral blood mononuclear cells. Human Immunology 66(2):95-103.

Hodgkin PD, Chin SH, Bartell G, Mamchak A, Doherty K, Lyons AB, Hasbold J. 1997. The importance of efficacy and partial agonism in evaluating models of $B$ lymphocyte activation. International Reviews of Immunology 15(1-2):101-127 DOI 10.3109/08830189709068173.

Kay JE. 1991. Mechanisms of T lymphocyte activation. Immunology Letters 29(12):51-54 DOI 10.1016/0165-2478(91)90198-J.

Lee JH, Lee JE, Kahng JY, Kim SH, Park JS, Yoon SJ, Um JY, Kim WK, Lee JK, Park J, Kim EH, Lee JH, Lee JH, Chung WS, Ju YS, Park SH, Chang JH, Kang SG, Lee JH. 2018. Human glioblastoma arises from subventricular zone cells with low-level driver mutations. Nature 560(7717):243-247 DOI 10.1038/s41586-018-0389-3.

Lee Y, Scheck AC, Cloughesy TF, Lai A, Dong J, Farooqi HK, Liau LM, Horvath S, Mischel PS, Nelson SF. 2008. Gene expression analysis of glioblastomas identifies the major molecular basis for the prognostic benefit of younger age. BMC Medical Genomics 1:52 DOI 10.1186/1755-8794-1-52.

Li B, Cui M, Diehn Y, Li R. 2017. Development and validation of an individualized immune prognostic signature in early-stage nonsquamous non-small cell lung cancer. JAMA Oncology 3(11):1529-1537 DOI 10.1001/jamaoncol.2017.1609.

Liang P, Chai H, Zhao Y, Wang G. 2020. Predictive analyses of prognostic-related immune genes and immune infiltrates for glioblastoma. Diagnostics 10(3)177 DOI 10.3390/diagnostics10030177.

Liang Y, Diehn M, Watson N, Bollen AW, Aldape KD, Nicholas MK, Lamborn KR, Berger MS, Botstein D, Brown PO, Israel MA. 2005. Gene expression profiling reveals molecularly and clinically distinct subtypes of glioblastoma multiforme. Proceedings of the National Academy of Sciences of the United States of America 102(16):5814-5819 DOI 10.1073/pnas.0402870102.

Newman AM, Liu CL, Green MR, Gentles AJ, Feng W, Xu Y, Hoang CD, Diehn M, Alizadeh AA. 2015. Robust enumeration of cell subsets from tissue expression profiles. Nature Methods 12(5):453-457 DOI 10.1038/nmeth.3337. 
Niess JH, Klaus J, Stephani J, Pfluger C, Degenkolb N, Spaniol U, Mayer B, Lahr G, von Boyen GB. 2012. NOD2 polymorphism predicts response to treatment in Crohn's disease-first steps to a personalized therapy. Digestive Diseases and Sciences 57(4):879-886 DOI 10.1007/s10620-011-1977-3.

Pencina MJ, D'Agostino RB. 2004. Overall C as a measure of discrimination in survival analysis: model specific population value and confidence interval estimation. Statistics in Medicine 23(13):2109-2123 DOI 10.1002/sim.1802.

Reuss DE, Mamatjan Y, Schrimpf D, Capper D, Hovestadt V, Kratz A, Sahm F, Koelsche C, Korshunov A, Olar A, Hartmann C, Reijneveld JC, Wesseling P, Unterberg A, Platten M, Wick W, Herold-Mende C, Aldape K, von Deimling A. 2015. IDH mutant diffuse and anaplastic astrocytomas have similar age at presentation and little difference in survival: a grading problem for WHO. Acta Neuropathologica 129(6):867-873 DOI 10.1007/s00401-015-1438-8.

Richter J, Schlesner M, Hoffmann S, Kreuz M, Leich E, Burkhardt B, Rosolowski M, Ammerpohl O, Wagener R, Bernhart SH, Lenze D, Szczepanowski M, Paulsen M, Lipinski S, Russell RB, Adam-Klages S, Apic G, Claviez A, Hasenclever D, Hovestadt V, Hornig N, Korbel JO, Kube D, Langenberger D, Lawerenz C, Lisfeld J, Meyer K, Picelli S, Pischimarov J, Radlwimmer B, Rausch T, Rohde M, Schilhabel M, Scholtysik R, Spang R, Trautmann H, Zenz T, Borkhardt A, Drexler HG, Moller P, MacLeod RA, Pott C, Schreiber S, Trumper L, Loeffler M, Stadler PF, Lichter P, Eils R, Kuppers R, Hummel M, Klapper W, Rosenstiel P, Rosenwald A, Brors B, Siebert R, Project IM-S. 2012. Recurrent mutation of the ID3 gene in Burkitt lymphoma identified by integrated genome, exome and transcriptome sequencing. Nature Genetics 44(12):1316-1320 DOI 10.1038/ng.2469.

Sakata-Yanagimoto M, Enami T, Yokoyama Y, Chiba S. 2014. Disease-specific mutations in mature lymphoid neoplasms: recent advances. Cancer Science 105(6):623-629 DOI 10.1111/cas.12408.

Saleh RR, Peinado P, Fuentes-Antras J, Perez-Segura P, Pandiella A, Amir E, Ocana A. 2019. Prognostic value of lymphocyte-activation gene 3 (LAG3) in cancer: a metaanalysis. Frontiers in Oncology 9:1040 DOI 10.3389/fonc.2019.01040.

Sato H, Jeggo PA, Shibata A. 2019. Regulation of programmed death-ligand 1 expression in response to DNA damage in cancer cells: implications for precision medicine. Cancer Science 110(11):3415-3423 DOI 10.1111/cas.14197.

Schmitz R, Young RM, Ceribelli M, Jhavar S, Xiao W, Zhang M, Wright G, Shaffer AL, Hodson DJ, Buras E, Liu X, Powell J, Yang Y, Xu W, Zhao H, Kohlhammer H, Rosenwald A, Kluin P, Muller-Hermelink HK, Ott G, Gascoyne RD, Connors JM, Rimsza LM, Campo E, Jaffe ES, Delabie J, Smeland EB, Ogwang MD, Reynolds SJ, Fisher RI, Braziel RM, Tubbs RR, Cook JR, Weisenburger DD, Chan WC, Pittaluga S, Wilson W, Waldmann TA, Rowe M, Mbulaiteye SM, Rickinson AB, Staudt LM. 2012. Burkitt lymphoma pathogenesis and therapeutic targets from structural and functional genomics. Nature 490(7418):116-120 DOI 10.1038/nature11378. 
Smart SK, Vasileiadi E, Wang X, De Ryckere D, Graham DK. 2018. The emerging role of TYRO3 as a therapeutic target in cancer. Cancer 10(12):474

DOI 10.3390/cancers10120474.

Wang Y, Dong Q, Xuan H, Zhao L, Qin T, Zhang Q. 2018. Lymphocyte-activation gene3 expression and prognostic value in neoadjuvant-treated triple-negative breast cancer. Journal of Breast Cancer 21(2):124-133 DOI 10.4048/jbc.2018.21.2.124.

Ware CF. 2008. Targeting lymphocyte activation through the lymphotoxin and LIGHT pathways. Immunological Reviews 223:186-201

DOI 10.1111/j.1600-065X.2008.00629.x.

Wick W, Osswald A, Wick M, Winkler F. 2018. Treatment of glioblastoma in adults. Therapeutic Advances in Neurological Disorders 11:1756286418790452.

Wu M, Yuan X, Li Q, Liao H, Liu Z. 2019. Identification of a five-gene signature and establishment of a prognostic nomogram to predict progression-free interval of papillary thyroid carcinoma. Frontiers in Endocrinology 10:790 DOI 10.3389/fendo.2019.00790.

Yuan Q, Cai HQ, Zhong Y, Zhang MJ, Cheng ZJ, Hao JJ, Wang MR, Wan JH. 2019. Overexpression of IGFBP2 mRNA predicts poor survival in patients with glioblastoma. Bioscience Reports 39(6)BSR20190045 DOI 10.1042/BSR20190045.

Zanders ED, Svensson F, Bailey DS. 2019. Therapy for glioblastoma: is it working? Drug Discovery Today 24(5):1193-1201 DOI 10.1016/j.drudis.2019.03.008.

Zanello G, Goethel A, Forster K, Geddes K, Philpott DJ, Croitoru K. 2013. Nod2 activates NF-kB in CD4+ T cells but its expression is dispensable for T cell-induced colitis. PLOS ONE 8(12):e82623 DOI 10.1371/journal.pone.0082623. 